\title{
In eigener Sache: Veränderungen bei der „Klinischen Pädiatrie“
}

Die „Klinische Pädiatrie“ steht für eine eigenständige Pädiatrie und hat als anerkannte Fachzeitschrift ihren festen Platz im Fach. Mit dem vorliegenden Heft geht die „Klinische Pädiatrie“ (früher: Archiv Jugendheilkunde) in den 115. Jahrgang. Diese traditionsreiche Zeitschrift erhält fortan eine leicht veränderte Organisationsstruktur. Mit dem Ziel, das gesamte Fach der Pädiatrie noch kompetenter darzustellen, haben wir das Herausgebergremium erweitert. So wirken neben den langjährigen Herausgebern, Prof. Dr. G. Heimann und Prof. Dr. Dr. F.C. Sitzmann, bei der Einwerbung geeigneter Beiträge und dem Begutachtungs-Verfahren ab sofort sechs neue Herausgeber mit, die jeweils ein wesentliches Teilgebiet der Pädiatrie vertreten. Dies sind Prof. Dr. G. Alzen (Gießen), Prof. Dr. U. Göbel (Düsseldorf), Prof. Dr. L. Gortner (Gießen), Prof. Dr. T. Paul (Göttingen), Prof. Dr. H.-M. Straßburg (Würzburg) und Prof. Dr. R. Urbanek (Wien). Auf den folgenden zwei Seiten haben Sie Gelegenheit, die neuen Mitherausgeber in einer kurzen persönlichen Vorstellung kennen zu lernen.Auch mit dem erweiterten Herausgebergremium wird die „Klinische Pädiatrie“ als anspruchsvolles Forum für die wissenschaftliche Information der Kinderheilkunde und ihrer Grenzgebiete fortgeführt. Wenngleich primär als ein deutschsprachiges Journal konzipiert, wird unsere Zeitschrift, wie bisher, gerne auch englischsprachigen Beiträgen offen stehen. Manuskripte sind weiterhin bei Prof. F.C. Sitzmann und Prof. G. Heimann einzureichen.Die bewährte Gliederung in Übersichtsreferate, Originalarbeiten, „Visite“ und Kasuistiken wird beibehalten. Als Novum möchten wir unseren Autoren ab sofort zusätzlich anbieten, besonders wichtige Themen möglichst schnell in Form einer kurzen und knappen Originalarbeit zu publizieren. In Anlehnung an die „Rapid Communications“ nach anglo-amerikanischem Vorbild können geeignete Artikel unter der Rubrik „Aktuelle Mitteilung“ veröffentlicht werden. Für die eingereichten Manuskripte in dieser Sparte sollen alle Anstrengungen unternommen werden, eine Entscheidung über die Veröffentlichung innerhalb drei Wochen herbeizuführen und die Arbeit innerhalb eines verkürzten Zeitraums zu veröffentlichen. Verständlicherweise sind bei einer positiven Entscheidung nur noch minimale Änderungen im Manuskript möglich. Nur Themen die dringend nach einer schnellen
Veröffentlichung verlangen, werden akzeptiert. Autoren werden daher gebeten, in einem kurzen Begleitbrief zu begründen, warum ihre Arbeit für die Rubrik „Aktuelle Mitteilung“ eingereicht wurde. Aufbau und Struktur des Manuskriptes entspricht einer Originalarbeit (max. 28000 Zeichen inklusive Referenzen). Maximal 15 Literaturangaben sind zulässig. Manuskripte, die als „Aktuelle Mitteilung“ abgelehnt wurden, können als reguläre Artikel wieder eingereicht werden.Wir wünschen Ihnen und uns, dass diese Neuerungen mit dazu beitragen können, dass die „Klinische Pädiatrie“ auch künftig auf eine breite Autoren- und Leserschaft zählen darf und ihren Lesern weiterhin aktuelle, vor allem hilfreiche Informationen bieten wird.Herausgeber und Verlag

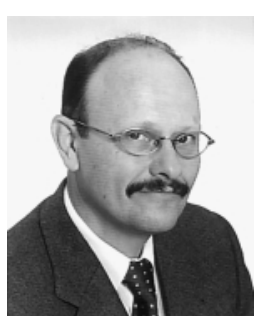

Prof. Dr. med. Gerhard Alzen, geboren 1948 in Wiesbaden, absolvierte sein Medizinstudium an den Universitäten in Frankfurt und Mainz. Nach der Approbation und Promotion 1977 arbeitete er als wissenschaftlicher Assistent an der Univ. Kinderklinik Mainz und erlangte 1982 die Facharztanerkennung für Pädiatrie. Anschließend begann er eine radiologische Weiterbildung am Institut für Klinische Strahlenkunde Mainz und wechselte 1985 an die Klinik für Radiologische Diagnostik der RWTH Aachen, wo er 1986 die Facharztanerkennung erwarb. Von 1987 bis 1992 war er Oberarzt und anschließend leitender Oberarzt der Klinik. 1990 erwarb er die Teilgebietsbezeichnung Kinderradiologie und habilitierte im Fach Radiologische Diagnostik mit einer Arbeit über die Strahlenexposition bei der Digitalen Substraktionsan- 
giographie im Kindesalter. Seit 1996 ist er Direktor der Abteilung Kinderradiologie des Klinikums der Justus-Liebig-Universität Gießen. Seine klinischen und wissenschaftlichen Schwerpunkte sind die Schnittbilddiagostik angeborener Erkrankungen und Interventionen im Kindesalter.

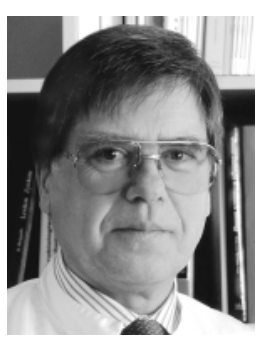

Prof. Dr. med. Ulrich Göbel,

geboren am 22. Juli 1941 in Dortmund, absolvierte sein Studium der Medizin in Köln und Düsseldorf. Vom 1969 bis Anfang 1973 erfolgte die Weiterbildung zum Facharzt für Kinderheilkunde am heutigen Zentrum für Kinder- und Jugendmedizin in Düsseldorf. 1974 habilitierte er sich zu dem Thema „ABO-Inkompatibilität und Ikterus neonatorum“. 1977 Ernennung zum C3-Professor an der Universität Düsseldorf und Beauftragung mit der Leitung der Klinik für Pädiatrische Hämatologie und Onkologie. Seit 1983 Studienleiter der kooperativen Therapieoptimierungsstudie für maligne Keimzelltumoren (MAKEI) der Gesellschaft für Pädiatrische Onkologie und Hämatologie und seit 2001 auch für maligne Hodentumoren (MAHO). 1991 wurde er in den Medizinischen Beirat der Deutschen Krebshilfe berufen und später in die gemeinsame Leitkommission der Deutschen Krebsgesellschaft und der Deutschen Krebshilfe sowie das ClearingHouse Onkologie delegiert. Gründungsmitglied der Kommission für Somatische Gentherapie des Wissenschaftlichen Beirates der Bundesärztekammer sowie des Koordinierungszentrums für Klinische Studien der Universität Düsseldorf. Mit Datum vom 15. November 2002 ist er zum Geschäftsführenden Direktor des Zentrums für Kinder- und Jugendmedizin der Universität Düsseldorf benannt worden.

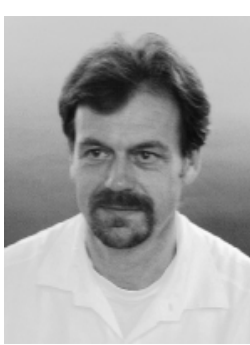

\section{Prof. Dr. med. Ludwig Gortner}

wurde am 13. November 1950 in Pirmasens in Rheinland-Pfalz geboren. Er absolvierte das Studium der Medizin an der Johann Wolfgang Goethe-Universität Frankfurt/ Main und arbeitete nach seiner Approbation und Promotion 1978 als wissenschaftlicher Assistent am dortigen Zentrum für Kinderheilkunde. Ab Mai 1983 wechselte er als Oberarzt an die Universitäts-Kinderklinik Ulm, wo er im gleichen Jahr seine Facharztprüfung absolvierte und 1990 seine Habilitation abschloss. Nach seiner Ernennung als C3-Professor an der Medizinischen Universität zu Lübeck in 1991 übernahm er 1995 die Funktion des stellvertretenden Direktors der Klinik für Pädiatrie der Medizinischen Universität zu Lübeck. Im September 1998 wurde er zum C4-Professor an die Kinderklinik der JustusLiebig Universität Gießen berufen und ist dort seit Januar 2001 Geschäftsführender Direktor des Zentrums für Kinderheilkunde und Jugendmedizin.

\section{Prof. Dr. med. Thomas Paul}

wurde 1957 in Braunschweig geboren. Sein Studium der Medizin absolvierte er zwischen 1976 und 1982 an der Medizinischen Hochschule Hannover (MHH). Nach Approbation und Promotion sowie Grundwehrdienst arbeitete er zunächst als Praxisassistent in einer internistischen Praxis. Von 1984-1990 Weiterbildung zum Arzt für Kinderheilkunde an der Kinderklinik der MHH. 1988 Aufenthalt am Texas Children's Hospital in Houston, Texas, als Ausbildungsstipendiat der DFG. Von 1991 bis 2001 war er als Oberarzt der Abteilung Kinderheilkunde III, Pädiatrische Kardiologie und Intensivmedizin, in der Kinderklinik der MHH tätig, ab 1996 auch als Leitender Oberarzt. 1995 Habilitation für das Fach Kinderheilkunde sowie Anerkennung als Facharzt für Kinderheilkunde - Kinderkardiologie. 1998 Anerkennung der zusätzlichen Weiterbildung in der Speziellen Pädiatrischen Intensivmedizin. 1999 Verleihung des Titels eines Außerplanmäßigen Professors für Kinderheilkunde durch die MHH. Vom Mai 2001 bis September 2002 Associate Professor of Pediatrics an der Medical University of South Carolina, Charleston, South Carolina. Im Oktober 2002 folgte er dem Ruf auf die Universitätsprofessur für Pädiatrische Kardiologie und Intensivmedizin an der Georg-August-Universität Göttingen.

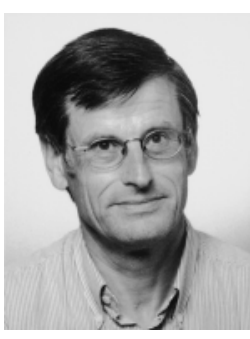

\section{Prof. Dr. med. Hans Michael Straßburg} wurde 1948 in Büchenbeuren/Hunsrück geboren. Von 1968 bis 1972 studierte er Humanmedizin in Tübingen, Wien und München und promovierte mit einer klinischneurophysiologischen Arbeit. Nach der Medizinalassistentenzeit klinische Tätigkeit in der Erwachsenen-Neurologie und -Psychiatrie sowie DFG-Ausbildungsstipendium im Institut für klinische Neurophysiologie Freiburg unter Prof. R. Jung. Ab 1976 Weiterbildung zum Facharzt für Kinderheilkunde in der Universitäts-Kinderklinik Freiburg, 1983 Funktionsoberarzt mit Schwerpunkt Neuropädiatrie, 1987 Habilitation über „Morphologie und Pathophysiologie perinataler Zerebralschäden“. 1988/1989 Gastprofessur am Institut für Sozialpädiatrie im Kinderzentrum München. Seit 1991 Professor für Kinderheilkunde mit Schwerpunkt Neuropädiatrie an der Universitäts-Kinderklinik Würzburg, außerdem Ärztlicher Leiter des seit 1992 bestehenden Sozialpädiatrischen Zentrums „Frühdiagnosezentrum".

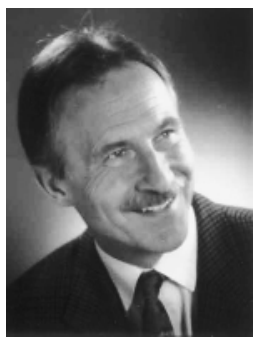

Prof. Dr. med. Radvan Urbanek,

Jahrgang 1943, absolvierte sein Medizinstudium an der Karls-Universität Prag. Nach der Approbation 1972 erhielt er die Facharztanerkennung 1995 an der Universitätsklinik für Kinderheilkunde in Freiburg/ Breisgau, wo er eine breite Ausbildung bekam. 1980 habilitierte er im Fach Pädiatrie und in den Jahren 1983/84 arbeitete er am Guy's Hospital in London (GB) als Stipendiat der Deutschen Forschungsgemeinschaft. 1990 wurde er als ordentlicher Professor an die Universität Wien berufen, wo er seither als Leiter der Abteilung für Allgemeine Pädiatrie tätig ist. Seine Interessengebiete und wissenschaftliche Schwerpunkte umfassen Allergologie, Immunologie sowie die pädiatrische Pulmologie. 